\title{
The Turbulent Heating Rate in Strong MHD Turbulence with Nonzero Cross Helicity
}

\author{
Benjamin D. G. Chandran \\ benjamin. chandran@unh.edu \\ Space Science Center and Department of Physics, University of New Hampshire \\ Eliot Quataert \\ elioteastro.berkeley.edu \\ Department of Astronomy, University of California, Berkeley \\ Gregory G. Howes \\ gregory-howes@uiowa.edu \\ Department of Physics \& Astronomy, University of Iowa \\ Joseph V. Hollweg \\ joe.hollweg@unh.edu \\ Space Science Center and Department of Physics, University of New Hampshire \\ William Dorland \\ bdorland@umd.edu \\ Department of Physics, University of Maryland
}

\begin{abstract}
Different results for the cascade power $\varepsilon$ in strong, incompressible MHD turbulence with nonzero cross helicity appear in the literature. In this paper, we discuss the conditions under which these different results are valid. Our conclusions can be expressed in terms of the density $\rho$, the rms amplitudes $z^{+}$and $z^{-}$of Alfvénic fluctuations propagating parallel and anti-parallel to the background magnetic field $\mathbf{B}_{0}$, and the correlation length (outer scale) measured perpendicular to $\mathbf{B}_{0}$, denoted $L_{\perp}$. We argue that if $z^{+} \gg z^{-}$and if the $z^{-}$fluctuations are sustained by the reflection of $z^{+}$fluctuations in a strong background magnetic field, then $\varepsilon \sim \rho\left(z^{+}\right)^{2} z^{-} / L_{\perp}$ as in
\end{abstract}


previous studies by Hossain, Matthaeus, Dmitruk, Lithwick, Goldreich, Sridhar, and others. On the other hand, if the minority wave type $\left(z^{-}\right)$is sustained by some form of forcing that is uncorrelated with or only weakly correlated with the $z^{+}$fluctuations, then $\varepsilon$ can be much less than $\rho\left(z^{+}\right)^{2} z^{-} / L_{\perp}$, as in previous studies by Dobrowolny, Lazarian, Chandran and others. The mechanism for generating the minority wave type strongly affects the cascade power because it controls the coherence time for interactions between oppositely directed wave packets at the outer scale.

Subject headings: turbulence — magnetic fields — magnetohydrodynamics — solar wind - solar corona — solar flares

\section{Introduction}

Turbulence plays an important role in numerous astrophysical plasmas, from the solar corona and solar wind (Coleman 1968; Belcher \& Davis 1971; Tu \& Marsch 1995; Goldstein, Matthaeus, \& Roberts 1995) to star-forming molecular clouds and clusters of galaxies (Boldyrev, Nordlund, \& Padoan 2002; Narayan \& Medvedev 2001; Cho \& Lazarian 2003; Elmegreen \& Scalo 2004). In general, astrophysical turbulence is compressible, and the compressive component of the turbulence may play an important role in processes such as ion heating and the scattering of energetic particles (Li \& Habbal 2001; Yan \& Lazarian 2004; Chandran 2005, 2008a). However, in this paper we focus on the non-compressive component of the turbulence, which generally dominates the energy at scales smaller than the collisional mean free path, since (non-compressive) Alfvén waves experience negligible linear collisionless damping at wavelengths $\lambda$ much larger than the proton gyroradius $r_{i}$ and frequencies $\omega$ much smaller than the proton cyclotron frequency $\Omega_{i}$ (Barnes 1966).

For $\lambda \gg r_{\mathrm{i}}$ and $\omega \ll \Omega_{i}$, the non-compressive component of the turbulence can be modeled using incompressible magnetohydrodynamics (MHD), even in a low-collisionality plasma. [See Howes et al (2008) and Schekochihin et al (2009) for recent discussions of this point.] In incompressible MHD, the fluctuating velocity and magnetic fields can be decomposed into fluctuations propagating parallel and anti-parallel to the background magnetic field $\mathbf{B}_{0}$, denoted $z^{+}$and $z^{-}$respectively. When the energies in $z^{+}$and $z^{-}$fluctuations differ, the turbulence has nonzero cross helicity 1 and is referred to as "imbalanced."

Turbulence in the solar corona and inner solar wind is imbalanced, with an excess of Alfvénic fluctuations propagating away from the Sun (Roberts et al 1987; Grappin et al 1990; Bavassano

\footnotetext{
${ }^{1}$ The cross helicity is defined as $\int d^{3} x \mathbf{v} \cdot \mathbf{B}$, where $\mathbf{v}$ is the velocity and $\mathbf{B}$ is the magnetic field.
} 
et al 2000a, 2000b; Bruno \& Carbone 2005). In order to model the heating of the solar corona and solar wind, it is of interest to determine the turbulent heating rate or cascade power in imbalanced MHD turbulence. Different expressions for this cascade power have appeared in the literature (see, e.g., Dobrowolny, Mangeney, \& Veltri 1980; Hossain et al 1995; Lithwick, Goldreich, \& Sridhar 2007; Beresnyak \& Lazarian 2008; Chandran 2008b). Our goal in this paper is to clarify the conditions under which these different expressions are valid. We focus on the difference between situations in which the minority Alfvén wave type (which we take to be $z^{-}$) is sustained by the reflection of $z^{+}$fluctuations and situations in which the $z^{-}$fluctuations are sustained by some form of forcing. Although a number of studies have addressed the effects of cross helicity on the inertialrange power spectra of $z^{+}$and $z^{-}$fluctuations (e.g., Grappin, Pouquet, \& Léorat 1983; Lithwick et al 2007; Beresnyak \& Lazarian 2008, 2009; Chandran 2008b; Perez \& Boldyrev 2009; Podesta $\&$ Bhattacharjee 2009), we do not address the inertial-range power spectra in this paper.

\section{Theoretical Framework}

We consider Alfvénic (i.e., transverse and non-compressive) fluctuations in a stationary, inhomogeneous medium, with background density $\rho$ and background magnetic and velocity fields $\mathbf{B}_{0}$ and $\mathbf{u}=u \mathbf{B}_{0} / B_{0}$. The velocity and magnetic-field fluctuations $\delta \mathbf{v}$ and $\delta \mathbf{B}$ can be written in terms of the Elsasser variables

$$
\mathbf{z}^{ \pm}=\delta \mathbf{v} \mp \frac{\delta \mathbf{B}}{\sqrt{4 \pi \rho}} .
$$

The equations for $\mathbf{z}^{ \pm}$are (Velli 1993, Verdini \& Velli 2007)

$$
\frac{\partial \mathbf{z}^{ \pm}}{\partial t}+\left(\mathbf{u} \pm \mathbf{v}_{\mathrm{A}}\right) \cdot \nabla \mathbf{z}^{ \pm}+\mathbf{z}^{\mp} \cdot \nabla\left(\mathbf{u} \mp \mathbf{v}_{\mathrm{A}}\right) \mp \frac{1}{2}\left(\mathbf{z}^{ \pm}-\mathbf{z}^{\mp}\right)\left(\nabla \cdot \mathbf{v}_{\mathrm{A}} \mp \frac{1}{2} \nabla \cdot \mathbf{u}\right)=-\mathbf{z}^{\mp} \cdot \nabla \mathbf{z}^{ \pm}-\frac{\nabla p_{\text {tot }}}{\rho},
$$

where $\mathbf{v}_{\mathrm{A}}=\mathbf{B}_{0} / \sqrt{4 \pi \rho}$ and $p_{\text {tot }}=p+B^{2} / 8 \pi$ is the sum of the thermal and magnetic pressure. The second term on the left-hand side of equation (2) represents wave propagation at velocity $\pm \mathbf{v}_{\mathrm{A}}$ in the frame of the plasma. The third and fourth terms on the left-hand side give rise to wave reflection. The right-hand side of equation (2) contains the nonlinear terms. Nonlinear interactions arise only when oppositely propagating wave packets interact (Iroshnikov 1963; Kraichnan 1965). In this paper, we focus on turbulent fluctuations at the "outer scale" or energy-injection scale. We view these outer-scale fluctuations as a collection of $z^{+}$and $z^{-}$wave packets with correlations lengths $L_{\perp}$ and $L_{\|}^{ \pm}$perpendicular and parallel to $\mathbf{B}_{0}$, where for simplicity we have taken $L_{\perp}$ to be the same for both the $z^{+}$and $z^{-}$fluctuations.

The shearing of a $z^{ \pm}$wave packet at the outer scale is dominated by interactions with $z^{\mp}$ wave packets at the outer scale. Since we take the fluctuations to be Alfvénic in nature, $\mathbf{z}^{ \pm}$is 
perpendicular to $\mathbf{B}_{0}$, and the magnitude of the $\mathbf{z}^{\mp} \cdot \nabla \mathbf{z}^{ \pm}$term in equation (2) is $\sim z_{0}^{-} z_{0}^{+} / L_{\perp}$, where $z_{0}^{ \pm}$is the rms amplitude of the $z^{ \pm}$fluctuations at the outer scale. The rate of shearing of $z^{ \pm}$wave packets by $z^{\mp}$ wave packets at the outer scale is thus

$$
\omega_{\text {shear }}^{\mp} \sim \frac{z_{0}^{\mp}}{L_{\perp}} .
$$

Nonlinear interactions between $z^{+}$and $z^{-}$fluctuations can be thought of as "collisions" between oppositely directed wave packets. If we consider some point $\mathrm{P}$ moving with a $z^{ \pm}$wave packet at velocity $\pm v_{\mathrm{A}} \hat{z}$, the time required for this point to pass through an outer-scale $z^{\mp}$ wave packet is approximately

$$
t_{\text {coll }}^{\mp} \sim \frac{L_{\|}^{\mp}}{v_{\mathrm{A}}}
$$

(where we have ignored the factor of $1 / 2$ that results from the fact that the relative speed of point $\mathrm{P}$ and the moving $z^{\mp}$ wave packet is $2 v_{\mathrm{A}}$ ). We define the quantity

$$
\chi^{\mp}=\frac{z_{0}^{\mp} L_{\|}^{\mp}}{L_{\perp} v_{\mathrm{A}}} \sim \omega_{\text {shear }}^{\mp} t_{\text {coll }}^{\mp} .
$$

If $\chi^{\mp}<1$, then $\chi^{\mp}$ is approximately the fractional change in an outer-scale $z^{ \pm}$wave packet caused by shearing by outer-scale $z^{\mp}$ fluctuations during a single "collision" of duration $t_{\text {coll }}^{\mp}$. If $\chi^{\mp} \gtrsim 1$, then an outer-scale $z^{ \pm}$wave packet undergoes a fractional change of order unity in a time $\sim L_{\perp} / z^{\mp}$, which is $\lesssim t_{\text {coll }}^{\mp}$.

We take $z^{+}$to be the dominant wave type, and assume that

$$
z_{0}^{-} \ll z_{0}^{+} \lesssim v_{A}
$$

which implies that $\delta B \lesssim B_{0}$. Throughout this paper, we focus on the strong-turbulence limit, in which

$$
\chi^{+} \gtrsim 1
$$

We note that the conditions $\chi^{+} \gtrsim 1$ and $\delta B \ll B_{0}$ are compatible if $L_{\perp} / L_{\|}^{+}$is sufficiently small.

\section{Forced Turbulence}

If a magnetized plasma is physically stirred by a set of objects distributed throughout its volume, and if the motions of the different stirring objects are uncorrelated, then this stirring will excite both $z^{+}$and $z^{-}$waves, with comparable power going into each wave type. If this stirring is the primary mechanism for exciting Alfvénic fluctuations, then the resulting turbulence will be 
approximately balanced, with little cross helicity. However, volumetric forcing can play an important role in imbalanced turbulence if, in addition to the forcing, a large flux of the dominant $z^{+}$ fluctuations is launched into the plasma from the plasma boundary. If the forcing is weak, then $z_{0}^{-}$ will be small, the cascade time of the $z^{+}$fluctuations will be large, and the $z^{+}$fluctuations injected at the plasma boundary will propagate a large distance into the plasma before their energy cascades. The role of forcing is then different for $z^{+}$and $z^{-}$. Within a certain distance of the boundary (see section 3.1), forcing makes only a small contribution to the energy of the $z^{+}$fluctuations, but is the mechanism that sustains the $z^{-}$fluctuations. We note that if $z^{+}$fluctuations satisfying equation (7) are launched into the plasma from the plasma boundary, and if $z^{-}$energy is not continually injected into the system throughout its volume, then $z^{-}$energy will decay on the short time scale $L_{\perp} / z_{0}^{+}$, leaving the system in a maximally aligned state 2 with vanishing cascade power (Dobrowolny et al 1980; Matthaeus \& Montgomery 1980; Pouquet, Sulem, \& Meneguzzi 1988).

We model the forcing of $z^{ \pm}$fluctuations as a stochastic source term $\mathbf{F}(\mathbf{x}, t)$ added to right-hand side of equation (2). For simplicity, we take the spatial variation of $\mathbf{F}$ in directions perpendicular to $\mathbf{B}_{0}$ to be characterized by a single scale length (correlation length), which we set equal to the perpendicular correlation length of the outer-scale $z^{+}$fluctuations, $L_{\perp}$. We also take the spatial variation of $\mathbf{F}$ along $\mathbf{B}_{0}$ to be characterized by a single scale length, denoted $L_{\|, f}$, which can differ from $L_{\|}^{+}$. We assume that the (stochastic) time variation of $\mathbf{F}(\mathbf{x}, t)$ is characterized by the single time scale $L_{\|, \mathrm{f}} / v_{\mathrm{A}}$.

The properties of outer-scale $z^{-}$wave packets are determined by both (1) the way in which the $z^{-}$fluctuations are sheared by $z^{+}$fluctuations and (2) the source term. We discuss these two mechanisms in turn and their consequences for the correlation time of the shearing that is applied by $z^{-}$wave packets and experienced by a single, moving $z^{+}$wave packet. Because of equation (7), $z^{-}$fluctuations can only propagate a distance $\lesssim L_{\|}^{+}$before their energy cascades to smaller scales. As a result, if we consider the outer-scale $z^{+}$wave packet labeled "A" in Figure 1, the outer-scale $z^{-}$fluctuations encountered by wave packet $A$ are sheared primarily by wave packet $A$ and the outer-scale wave packet immediately "ahead" of wave packet A, which is labeled wave packet B in Figure 1. Since wave packet B remains in front of wave packet A as the two wave packets propagate along the magnetic field, the shearing experienced by the outer-scale $z^{-}$fluctuations that are encountered by wave packet A remains coherent throughout the cascade time of wave packet A. On the other hand, the properties of the source term $\mathbf{F}$ in the region that contributes to the $z^{-}$fluctuations encountered by wave packet A decorrelates over a time scale $\sim L_{\|, f} / v_{\mathrm{A}}$, because of both the space and time dependence of $\mathbf{F}$. Thus, the coherence time for the shearing experienced

\footnotetext{
${ }^{2}$ When $\mathbf{z}^{-}=\delta \mathbf{v}+\delta \mathbf{B} / \sqrt{4 \pi \rho}=0$, the state is called "maximally aligned" because $\delta \mathbf{v}$ and $\delta \mathbf{B}$ are everywhere antiparallel.
} 
by the outer-scale $z^{+}$wave packets is 3

$$
t_{\text {cor }}^{-} \sim \frac{L_{\|, \mathrm{f}}}{v_{\mathrm{A}}} .
$$

We note that if $L_{\|, \mathrm{f}} \gg L_{\|}^{+}$, then because of equation (7) the shearing experienced by outer-scale $z^{-}$fluctuations will cause the $z^{-}$fluctuations to vary strongly over a distance $L_{\|}^{+}$along the field, causing $L_{\|}^{-}$to become comparable to $L_{\|}^{+}$. However, in this case, the shearing experienced by the $z^{+}$ fluctuations remains coherent for a time $L_{\|, \mathrm{f}} / v_{\mathrm{A}}$ rather than the collision time scale $t_{\text {coll }}^{-} \sim L_{\|}^{-} / v_{\mathrm{A}}$, for the reasons given above.

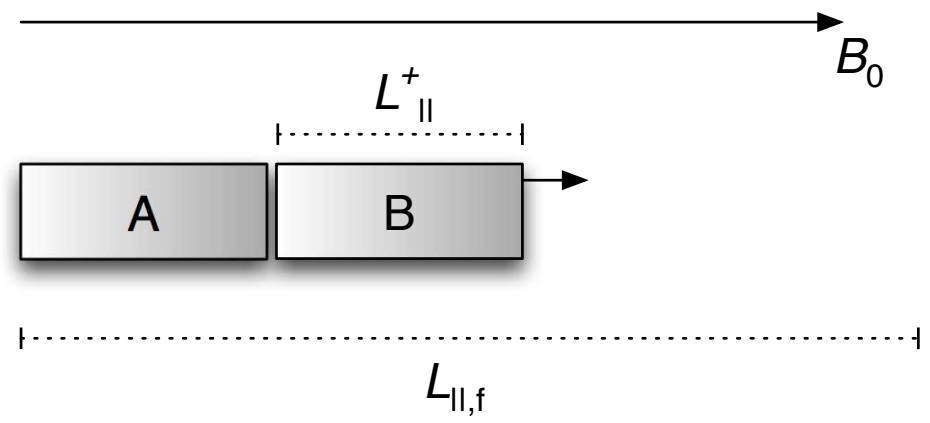

Fig. 1.- A and B represent two outer-scale $z^{+}$wave packets propagating along the background magnetic field $\mathbf{B}_{0}$. In this pictorial example, the parallel correlation length of the forcing term, $L_{\|, \mathrm{f}}$ is taken to be longer than the parallel correlation length of the outer-scale $z^{+}$wave packets, $L_{\|}^{+}$.

We define the quantity

$$
\hat{\chi}^{-}=\frac{z_{0}^{-} L_{\|, \mathrm{f}}}{v_{\mathrm{A}} L_{\perp}} .
$$

We first consider the case in which

$$
\hat{\chi}^{-}<1 \text {. }
$$

When equation (10) is satisfied, $\hat{\chi}^{-}$is approximately the fractional change in an outer-scale $z^{+}$ wave packet during a time $t_{\text {cor }}^{-}$. During successive time intervals of duration $t_{\text {cor }}^{-}$, the incremental changes in the $z^{+}$wave packet that are caused by the shearing of $z^{-}$fluctuations add like a random walk. Thus, $\left(\hat{\chi}^{-}\right)^{-2}$ time intervals of duration $t_{\text {cor }}^{-}$are required in order for the incremental changes in the $z^{+}$wave packet to accumulate to an order-unity fractional change. The cascade time for outer-scale $z^{+}$wave packets is then given by

$$
t_{\text {casc }}^{+} \sim \frac{L_{\perp}^{2} v_{\mathrm{A}}}{\left(z_{0}^{-}\right)^{2} L_{\|, \mathrm{f}}}
$$

\footnotetext{
${ }^{3}$ Equation (8) is valid unless $L_{\|, \mathrm{f}} / v_{\mathrm{A}}$ exceeds the $z^{+}$cascade time $t_{\text {casc }}^{+}$, in which case $t_{\text {cor }}^{-} \sim t_{\text {casc }}^{+}$, and the cascade time and cascade power are given by equations (14), (16), and (17).
} 
The cascade power in $z^{+}$fluctuations, $\varepsilon^{+} \sim \rho\left(z_{0}^{+}\right)^{2} / t_{\text {casc }}^{+}$, is then (Beresnyak \& Lazarian 2008, Chandran 2008b)

$$
\varepsilon^{+} \sim \frac{\rho\left(z_{0}^{+}\right)^{2}\left(z_{0}^{-}\right)^{2} L_{\|, f}}{v_{\mathrm{A}} L_{\perp}^{2}} .
$$

If we set $L_{\perp}=L_{\|, \mathrm{f}}$, then equation (12) reduces to the result of Dobrowolny et al (1980) for isotropic turbulence. In the case that $L_{\|, \mathrm{f}} \sim L_{\|}^{+}$and $\chi^{+} \sim 1$, we can rewrite equation (12) as (Chandran 2008b)

$$
\varepsilon^{+} \sim \frac{\rho z_{0}^{+}\left(z_{0}^{-}\right)^{2}}{L_{\perp}}
$$

Because of equation (7), the cascade time for $z^{-}$fluctuations is simply $t_{\text {casc }}^{-} \sim L_{\perp} / z_{0}^{+}$, and the cascade power in $z^{-}$fluctuations is

$$
\varepsilon^{-} \sim \frac{\rho z_{0}^{+}\left(z_{0}^{-}\right)^{2}}{L_{\perp}},
$$

comparable to the value of $\varepsilon^{+}$in equation (13). 4

If, instead of equation (10),

$$
\hat{\chi}^{-}>1
$$

then the shearing experienced by outer-scale $z^{+}$wave packets remains coherent throughout their cascade time,

$$
t_{\mathrm{casc}}^{+} \sim \frac{L_{\perp}}{z_{0}^{-}}
$$

and

$$
\varepsilon^{+} \sim \frac{\rho\left(z_{0}^{+}\right)^{2} z_{0}^{-}}{L_{\perp}},
$$

while $\varepsilon^{-}$is still given by equation (14). Turbulent heating rates of this form were previously obtained by Hossain et al (1995) on the basis of numerical simulations of decaying MHD turbulence, and by Lithwick et al (2007) on the basis of theoretical arguments.

\footnotetext{
${ }^{4}$ In the weak-turbulence limit $\chi^{-} \ll \chi^{+} \ll 1$, both $\varepsilon^{+}$and $\varepsilon^{-}$scale in the same way with $z_{0}^{ \pm}, L_{\perp}$, and $L_{\|}$[in particular, $\varepsilon^{ \pm} \propto \rho\left(z_{0}^{+}\right)^{2}\left(z_{0}^{-}\right)^{2} L_{\|} /\left(v_{A} L_{\perp}^{2}\right)$ (Lithwick \& Goldreich 2003)]. However, there is an additional dependence of $\varepsilon^{ \pm}$on the inertial-range spectral indices, so that the fluctuation type $\left(z^{+}\right.$or $\left.z^{-}\right)$with the steeper inertial-range power spectrum has the larger cascade power. It was shown analytically by Galtier et al (2000) and Lithwick \& Goldreich (2003) that this dependence becomes very strong as the spectral indices approach the limiting values at which the assumption of local interactions breaks down, so that the ratio $\varepsilon^{+} / \varepsilon^{-}$can take on any value. In the strong turbulence limit $\chi^{-} \ll \chi^{+} \sim 1, \varepsilon^{+}$and $\varepsilon^{-}$also scale in the same way with $z_{0}^{ \pm}$and $L_{\perp}$ when $L_{\|, \mathrm{f}} \sim L_{\|}^{+}$and $\hat{\chi}^{-}<$ 1 , as shown in equations (13) and (14). However, if $\varepsilon^{+}$and $\varepsilon^{-}$depend on the inertial-range spectral indices in the strong turbulence case as they do in the weak-turbulence limit, then additional coefficients (not necessarily of order unity) need to be included in equations (13) and (14). The presence of such coefficients would allow for the possibility of forced, steady-state, strongly turbulent flows in which $\varepsilon^{+} / \varepsilon^{-}$differs significantly from unity, even when the assumptions leading to equations (13) and (14) are valid (i.e., $\chi^{+} \simeq 1, \hat{\chi}^{-}<1$, and $L_{\|, \mathrm{f}} \sim L_{\|}^{+}$).
} 


\subsection{An Example of Forced, Imbalanced MHD Turbulence}

In this section, we consider a finite plasma that is stirred by a set of objects located throughout its volume. We take the dimension and spacing of these objects perpendicular to the magnetic field to be $L_{\perp}$, and we take the objects to be spaced by a distance $L_{\|}^{+}$along the magnetic field. We take the correlation time of the forcing to be $L_{\|}^{+} / v_{\mathrm{A}}$. In the absence of wave launching at the boundary, these stirring objects set up balanced MHD turbulence, in which the rms amplitudes of the $z^{+}$and $z^{-}$fluctuations are both equal to a quantity that we denote $\delta z$. We define

$$
\chi_{0}=\frac{L_{\|}^{+} \delta z}{L_{\perp} v_{\mathrm{A}}}
$$

and assume that

$$
\chi_{0} \ll 1 .
$$

Thus, in the absence of wave launching, the forcing would set up weak turbulence at the outer scale, with an outer-scale cascade time of $\left(\chi_{0}\right)^{-2} L_{\|}^{+} / \nu_{\mathrm{A}}$ and a cascade power of

$$
Q_{0} \sim \frac{\rho(\delta z)^{4} L_{\|}^{+}}{L_{\perp}^{2} v_{\mathrm{A}}}
$$

We now assume that $z^{+}$waves are launched into the plasma from one its boundaries (the "launching boundary") with correlation lengths $L_{\perp}$ and $L_{\|}^{+}$perpendicular and parallel to the magnetic field. We consider the region that is sufficiently close to the launching boundary that the amplitudes of the $z^{+}$fluctuations have not decayed very much from their values right next to the boundary. (The size of this region is estimated below.) We assume that within this region,

$$
\chi^{+} \simeq 1
$$

implying that $z_{0}^{+} \gg \delta z$. We also assume that the presence of the additional $z^{+}$waves does not substantially alter the rate at which energy is injected into either the $z^{+}$of $z^{-}$fluctuations 5 Since the cascade time for the $z^{-}$fluctuations is short, the rate at which energy is injected into $z^{-}$fluctuations will be in balance with the rate at which $z^{-}$energy cascades to small scales. Thus,

$$
Q_{0} \sim \frac{\rho\left(z_{0}^{-}\right)^{2} z_{0}^{+}}{L_{\perp}}
$$

\footnotetext{
${ }^{5}$ This assumption is plausible for the following reason. The net power per unit volume going into the $z^{ \pm}$fluctuations, denoted $Q^{ \pm}$, is approximately $\left\langle\mathbf{F} \cdot \mathbf{z}^{ \pm}\right\rangle$, where $\langle\ldots\rangle$ represents an ensemble average. The value of $\mathbf{z}^{ \pm}$can be thought of as consisting of two components, $\mathbf{z}^{ \pm}=\mathbf{z}_{1}^{ \pm}+\mathbf{z}_{2}^{ \pm}$, where $\mathbf{z}_{1}^{ \pm}$is correlated with $\mathbf{F}$, and $\mathbf{z}_{2}^{ \pm}$is not. A rough estimate of $Q^{ \pm}$is then $F_{\text {rms }} z_{1, \text { rms }}^{ \pm}$, where $F_{\text {rms }}$ and $z_{1, \text { rms }}^{ \pm}$are the rms amplitudes of $\mathbf{F}$ and $\mathbf{z}_{1}^{ \pm}$. Because the correlation time of $\mathbf{F}$ in our example is $L_{\|}^{+} / v_{\mathrm{A}}$, which is comparable to or shorter than the cascade time of each fluctuation type, $z_{1, \mathrm{rms}}^{ \pm} \sim F_{\mathrm{rms}} L_{\|}^{+} / v_{\mathrm{A}}$ for both wave types, so that $Q^{+} \sim Q^{-}$.
} 
Equations (20) through (22) then lead to the relation

$$
z_{0}^{-} \sim \frac{(\delta z)^{2}}{z_{0}^{+}},
$$

which illustrates how the enhanced level of $z^{+}$fluctuations reduces $z_{0}^{-}$relative to its value $(\delta z)$ in the absence of wave launching from the boundary.

Determining the rate at which $z^{+}$energy decays in this example is subtle. Equations (12) and (23) imply that

$$
\varepsilon^{+} \sim \frac{\rho(\delta z)^{4} L_{\|}^{+}}{L_{\perp}^{2} v_{\mathrm{A}}},
$$

which is comparable to the rate at which energy is injected by the forcing term. The question of whether the $z^{+}$energy increases or decreases with increasing distance from the launching boundary thus depends upon the undetermined factors of order unity in equations (20) and (24). To proceed, we conjecture that the $z^{+}$and $z^{-}$power spectra are "pinned" (i.e., equal) at the dissipation scale (Grappin et al 1983; Lithwick \& Goldreich 2003 — but see Perez \& Boldyrev 2009 and Beresnyak \& Lazarian 2009), and that there is a dimensionless prefactor in the expression for $\varepsilon^{+}$(or $\varepsilon^{-}$) that increases as the $z^{+}$(or $z^{-}$) inertial-range power spectrum becomes steeper, as in the case of imbalanced weak MHD turbulence (Galtier et al 2000; Lithwick \& Goldreich 2003). This implies that in the imbalanced case in which $z_{0}^{+} \gg z_{0}^{-}$, the value of $\varepsilon^{+}$will be somewhat larger than both $\varepsilon^{-}$ and $Q_{0}$, so that the $z^{+}$energy will decay in time, when viewed from a frame of reference moving with the $z^{+}$fluctuations at velocity $v_{\mathrm{A}} \hat{z}$. The time scale $t_{\text {casc }}^{+}$for this decay is given approximately by equation (11), with $L_{\|, \mathrm{f}}$ set equal to $L_{\|}^{+}$. In the plasma frame, the $z^{+}$energy then decays over a distance of $L_{\text {decay }}^{+} \sim v_{\mathrm{A}} t_{\text {casc }}^{+}$, or $\left(\right.$since $\left.\chi^{+} \simeq 1\right)$

$$
L_{\text {decay }}^{+} \sim L_{\|}^{+} \chi_{0}^{-4} \text {. }
$$

At distances $\lesssim L_{\text {decay }}^{+}$from the launching boundary, the turbulence would then be highly imbalanced, with forcing sustaining the $z^{-}$fluctuations but making only a small contribution to the energy in $z^{+}$fluctuations. On the other hand, at distances $\gg L_{\text {decay }}^{+}$from the launching boundary the turbulence would become approximately balanced. If our conjecture above about the relation between the cascade power and inertial-range spectral index in strong imbalanced turbulence is incorrect, then other phenomenologies are possible.

\section{Reflection-Driven Turbulence in a Strong Background Magnetic Field}

In this section, we assume that the $z^{-}$wave packets are generated by reflection of the dominant $z^{+}$wave packets. The key difference from the last section is that the mechanism generating the 
$z^{-}$fluctuations is now strongly correlated with, and in fact determined by, the properties of the $z^{+}$ fluctuations. We begin by considering outer-scale $z^{+}$fluctuations that satisfy the "critical-balance condition" (Goldreich \& Sridhar 1995; Lithwick et al 2007),

$$
\chi^{+} \sim 1
$$

We consider the case $\chi^{+} \gg 1$ at the end of this section. Part of our motivation for considering outer scales satisfying equation (26) comes from the work of Cranmer \& van Ballegooijen (2005), who modeled the launching of Alfvén waves into the corona and solar wind from the Sun. They based their analysis on observations of photospheric motions and a model of wave propagation in the chromosphere, corona, and solar wind. They found that for heliocentric distances between 2 and 10 solar radii, the bulk of the wave power is at periods of $1-5$ minutes, with wave amplitudes such that equation (26) is approximately satisfied.

Equation (26) implies that the outer-scale $z^{-}$wave packets only travel a distance $\sim L_{\|}^{+}$before their energy cascades to smaller scales. Thus, the outer-scale $z^{-}$fluctuations present at an arbitrary point $\mathrm{P}$ originated within a distance $\sim L_{\|}^{+}$from $\mathrm{P}$. If we consider two outer-scale $z^{+}$wave packets A and B of length $L_{\|}^{+}$and width $L_{\perp}$, with A right behind B as in Figure 2, then the outer-scale $z^{-}$wave packets encountered by wave-packet A (denoted $B^{\prime}$ in Figure 2) will primarily be the $z^{-}$ field arising from the reflection of wave packet B. If we assume that the gradient scale lengths of the background medium are at least as long as the "damping length" $v_{\mathrm{A}} t_{\mathrm{casc}}^{+}$of the $z^{+}$wave packet, then the $z^{-}$wave packets encountered by wave packet A will continue to have the same form and structure until wave packet B changes. The shearing experienced by wave packet $A$ is thus coherent throughout its cascade time, rather than a random-walk-like accumulation of uncorrelated, smallamplitude distortions. The cascade time of wave packet $\mathrm{A}$ is therefore simply $\sim\left(\omega_{\text {shear }}^{-}\right)^{-1}$, or

$$
t_{\text {casc }}^{+} \sim \frac{L_{\perp}}{z_{0}^{-}},
$$

which implies that

$$
\varepsilon^{+} \sim \frac{\rho\left(z_{0}^{+}\right)^{2} z_{0}^{-}}{L_{\perp}}
$$

as in the studies of Hossain et al (1995) and Lithwick et al (2007).

Lithwick et al (2007) argued that in strong, imbalanced, MHD turbulence, the shearing felt by the $z^{+}$wave packets throughout the inertial range is coherent over a time that significantly exceeds the wave-packet "collision time" or crossing time, independent of the mechanism that generates the outer-scale fluctuations. Our arguments differ from theirs in two ways. First, our discussion is restricted to the outer-scale fluctuations. Second, we argue that equation (27) is valid when the minority $z^{-}$wave type is generated by wave reflection, but not when $z^{-}$is generated by forcing that is only weakly correlated with the $z^{+}$fluctuations (unless $\hat{\chi}^{-}>1$, as described in section 3 ). 


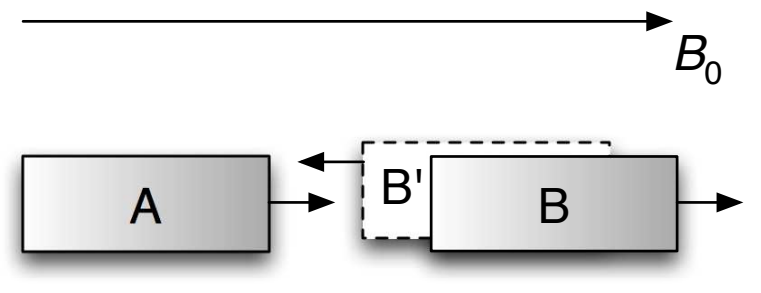

Fig. 2.- In strong, reflection-driven turbulence, the properties of the $z^{-}$wave packet $\left(\mathrm{B}^{\prime}\right)$ encountered by the $z^{+}$ wave packet on the left (A) are determined by the $z^{+}$wave packet on the right (B). The shearing of wave packet $\mathrm{A}$ by wave packet $\mathrm{B}^{\prime}$ thus remains coherent over a time comparable to the cascade time of wave packet $\mathrm{B}$.

For the waves launched into the solar corona by photospheric motions, it is typically argued that $L_{\perp} \sim 10^{9} \mathrm{~cm}$ at the coronal base, and that the Alfvén waves at this perpendicular scale have a broad frequency spectrum, extending up to wave periods of many hours (Dmitruk \& Matthaeus 2003, Cranmer \& van Ballegooijen 2005, Verdini \& Velli 2007). Although Cranmer \& van Ballegooijen (2005) find that the energy in outward Alfvén waves $\left(z^{+}\right.$in our discussion) in the corona is dominated by waves with periods of $\sim 1-5$ minutes, wave reflection is more efficient for lowfrequency waves. Thus, if nonlinear wave-wave interactions were neglected, then the inward wave packets would have a longer parallel correlation length than the outward waves.

We now explore the effects of frequency-dependent reflection, including the effects of nonlinear wave-wave interactions, by considering the limiting case in which the source term for inward $\left(z^{-}\right)$waves is invariant along the magnetic field. The parallel correlation length of the $z^{-}$fluctuations is given by the arguments of Lithwick et al (2007), as follows. We continue to assume the validity of equation (26), which is motivated for the coronal case by the results of Cranmer \& van Ballegooijen's (2005) model of wave launching and propagation. Equation (26) implies that a $z^{-}$ wave packet can only travel a distance of order $L_{\|}^{+}$before the wave packet is strongly distorted. Since the $z^{+}$fluctuations decorrelate over a distance $L_{\|}^{+}$along the magnetic field, the inward waves acquire a parallel correlation length of $L_{\|}^{+}$by interacting with the outward waves, even though the parallel correlation length of the source term for the inward waves is $\gg L_{\|}^{+}$.

Nevertheless, the coherence time for the shearing experienced by an outer-scale $z^{+}$wave packet remains much longer than $t_{\text {coll }}^{-}$. This can again be seen with the aid of Figure 2. Now, the source term for the inward waves is invariant along the magnetic field, but the properties of the $z^{-}$wave packet $\left(\mathrm{B}^{\prime}\right)$ encountered by the $z^{+}$wave packet $\mathrm{A}$ are determined by the way in which the $z^{+}$wave packet B shears the inward waves generated by the uniform source term. Since wave 
packet $\mathrm{A}$ and $\mathrm{B}$ remain adjacent as they propagate outward along the magnetic field, the $z^{-}$wave field encountered by wave packet A will remain approximately the same until wave packet B changes, i.e., for a time equal to the cascade time of the outward waves. Since the shearing of the $z^{+}$wave packets is coherent throughout their cascade time, their cascade time is again given by equation (27), and the cascade power for $z^{+}$fluctuations is again given by equation (28).

We now turn briefly to the case in which $\chi^{+} \gg 1$, with the $z^{-}$fluctuations generated by wave reflection. As in the case $\chi^{+} \sim 1$, the shearing experienced by outer-scale $z^{+}$wave packets remains coherent throughout their cascade time, and the cascade power in $z^{+}$fluctuations is again given by equation (28), as in the studies by Matthaeus et al (1999) and Dmitruk \& Matthaeus (2003).

Although we have focused on wave reflection in this section, similar arguments would apply to a compressible plasma in which the minority waves $\left(z^{-}\right)$are generated by the parametric decay of $z^{+}$waves (Galeev \& Oraevskii 1963; Sagdeev \& Galeev 1969; Viñas \& Goldstein 1991; Malara et al 2000, 2001; Del Zanna et al 2001). In this case, the $z^{-}$waves encountered by the $z^{+}$wave packet $\mathrm{A}$ in Figure 2 will again be determined primarily by the properties of the $z^{+}$wave packet B, which is immediately ahead of wave packet $A$, and the shearing experienced by wave packet $A$ will remain coherent throughout the cascade time of wave packet $\mathrm{A}$.

\section{Application to the Solar Corona and Solar Wind}

In the solar corona, the dominant source of low-frequency, Sunward-propagating, Alfvén waves is likely wave reflection (Matthaeus et al 1999; Dmitruk et al 2001, 2002). The anti-Sunward waves launched by photospheric motions have sufficiently large amplitudes and low frequencies that $\chi^{+} \gtrsim 1$ within the corona (Cranmer \& van Ballegooijen 2005; Cranmer, van Ballegooijen, \& Edgar 2007; Verdini \& Velli 2007). If these anti-Sunward waves have enough time to develop a turbulent power spectrum extending to the (perpendicular) dissipation scale before they propagate out of the corona to larger heliocentric distances, then the cascade power and turbulent heating rate in the corona are given by equation (28), as in the studies of Matthaeus et al (1999), Dmitruk \& Matthaeus (2003), Cranmer \& van Ballegooijen (2005), and Verdini \& Velli (2007). The application of equations (12) and (13) to the corona by Chandran (2008b) was incorrect. The use of the correct equation in models of the coronal is critical, because the energy of anti-Sunward waves dramatically exceeds the energy of Sunward waves in the corona (Cranmer \& van Ballegooijen 2005; Verdini \& Velli 2007). When the correct expressions for $\varepsilon^{+}$and $\varepsilon^{-}$are used in models of the turbulent heating of open-magnetic-field-line regions in the solar corona, the heating rate is ener-

getically sufficient to explain the acceleration of the fast solar wind for plausible assumptions about the amplitude and frequency spectrum of the waves launched from the Sun (Matthaeus et al 1999; Dmitruk et al 2002; Cranmer \& van Ballegooijen 2005; Verdini \& Velli 2007). On the other hand, 
if equations (12) and/or (13) were used, then the heating rate in these models would be much too small to explain the acceleration of the fast wind, because $z_{0}^{-} / z_{0}^{+}$is so small in the corona. [See Cranmer \& van Ballegooijen (2005) and Verdini \& Velli (2007).]

In the solar wind at low heliographic latitudes, the Sunward-propagating Alfvén waves may be driven primarily by instabilities resulting from shear in the solar-wind velocity (Roberts et al 1992; Breech et al 2008). It is not entirely clear how to model this process, but a reasonable first approximation is to treat the generation of Sunward waves by velocity-shear instabilities as a form of mechanical forcing that is only weakly correlated with the anti-Sunward waves. If we denote the parallel correlation length of this forcing as $L_{\|, f}$, and if $\hat{\chi}^{-}<1$, then the cascade power and turbulent heating rate are given by equation (12), which is a factor of $\hat{\chi}^{-}$smaller than the value in equation (28) (Beresnyak \& Lazarian 2008; Chandran 2008b). If $L_{\|, f} \sim L_{\|}^{+}$and $\chi^{+} \sim 1$, then the cascade power and turbulent heating rate are given by equation (13) (Chandran 2008b). The difference between the different expressions for the turbulent heating rate in reflection-driven turbulence and forced turbulence makes less of a difference in models of the solar wind at heliocentric distances of $1 \mathrm{AU}$ and beyond than in models of the corona, because $z_{0}^{-} / z_{0}^{+}$is not so small. Nevertheless, this difference may be significant when comparing model temperature profiles with observations, as shown in the recent study by $\mathrm{Ng}$ et al (2009).

\section{Conclusion}

In this paper, we have argued that the cascade power in strong, imbalanced, incompressible MHD turbulence depends upon the mechanism that sustains the minority Alfvén wave type $\left(z^{-}\right)$ at the outer scale. If the outer-scale $z^{-}$wave packets are sustained by the reflection of $z^{+}$wave packets, then the shearing of the outer-scale $z^{+}$wave packets remains coherent throughout their cascade time, a time that can greatly exceed the crossing time of two counter-propagating outerscale wave packets. On the other hand, if the outer-scale $z^{-}$wave packets are sustained by some form of forcing that is only weakly correlated with the $z^{+}$fluctuations, then the coherence time for the shearing of the outer-scale $z^{+}$wave packets is shorter than the cascade time, unless the parallel correlation length of the forcing function is very long (i.e., unless $\hat{\chi}^{-}>1$ ). The longer coherence time in reflection-driven turbulence enhances the cascade power in reflection-driven turbulence relative to forced turbulence with the same values of $z_{0}^{ \pm}, L_{\perp}$, and $L_{\|}^{ \pm}$.

We thank Marco Velli, Bill Matthaeus, Steve Cranmer, and the referee Yoram Lithwick for helpful discussions and comments. This work was supported in part by the the Center for Integrated Computation and Analysis of Reconnection and Turbulence (CICART) under DOE Grant DEFG02-07-ER46372, by NSF-DOE Grant AST-0613622, by NSF Grant ATM-0851005, and by 
NASA under Grants NNX07AP65G and NNX08AH52G. E. Quataert was supported in part by NSF-DOE Grant PHY-0812811 and by NSF Grant ATM-0752503.

\section{REFERENCES}

Barnes, A. 1966, Phys. Fluids, 9, 1483

Bavassano, B., Pietropaolo, E.,\& Bruno, R. 2000a, J. Geophys. Res., 105, 12697

Bavassano, B., Pietropaolo, E., \& Bruno, R. 2000b, J. Geophys. Res., 105, 15959

Belcher, J. W., \& Davis, L. 1971, J. Geophys. Res., 76, 3534

Beresnyak, A., \& Lazarian, A. 2008, ApJ, 682, 1070

Beresnyak, A., \& Lazarian, A. 2009, arXiv:0904.2574

Boldyrev, S., Nordlund, A., \& Padoan, P. 2002, Phys. Rev. Lett., 89, 031102

Breech, B., Matthaeus, W. H., Minnie, J., Bieber, J. W., Oughton, S., Smith, C. W., \& Isenberg, P. A. 2008, J. Geophys. Res., 113, A08105

Bruno, R., \& Carbone, V. 2005, Living Rev. Solar Phys., 2, 4 [Online article: cited Nov. 14, 2007, http://www.livingreviews.org/lrsp-2005-4

Chandran, B. D. G. 2005, Phys. Rev. Lett., 95, 265004

Chandran, B. D. G. 2008a, Phys. Rev. Lett., 101, 235004

Chandran, B. D. G. 2008b, ApJ, 685, 646

Cho, J., \& Lazarian, A. 2003, MNRAS, 345, 325

Coleman, P. J. 1968, ApJ, 153, 371

Cranmer, S. R. \& van Ballegooijen, A. A. 2005, ApJS, 156, 265

Cranmer, S. R., van Ballegooijen, A. A., \& Edgar, R. J. 2007, ApJS, 171, 520

Del Zanna, L., Velli, M., \& Londrillo, P. 2001, A\&A, 367, 705

Dmitruk, P., Milano, L. J., \& Matthaeus, W. H. 2001, ApJ, 548, 482

Dmitruk, P., Matthaeus, W. H., Milano, L. J., Oughton, S., Zank, G. P., \& Mullan, D. J. 2002, ApJ, 575,571

Dmitruk, P., \& Matthaeus, W. H. 2003, ApJ, 597, 1097

Dobrowolny, M., Mangeney, A., Veltri, P. L. 1980, Phys. Rev. Lett., 35, 144

Galeev, A. A., \& Oraevskii, V. N. 1963, Sov. Phys. Dokl., 7, 988 
Galtier, S., Nazarenko, S. V., Newell, A. C., \& Pouquet, A. 2000, J. Plasma Phys., 63, 447

Goldreich, P., \& Sridhar, S. 1995, ApJ, 438, 763

Goldreich, P., \& Sridhar, S. 1997, ApJ, 485, 680

Goldstein, B. E., Smith, E. J., Balogh, A., Horbury, T. S., Goldstein, M. L., \& Roberts, D. A. 1995, Geophys. Res. Lett., 22, 3393

Goldstein, M. L., Roberts, D. A., \& Matthaeus, W. H. 1995, Ann. Rev. Astron. Astrophys., 33, 283

Grappin, R., Pouquet, A., \& Léorat, J. 1983, A\&A, 126, 51

Grappin, R., Mangeney, A., \& Marsch, E. 1990, J. Geophys. Res., 95, 8197

Hossain, M., Gray, P. C., Pontius, D. H., Matthaeus, W. H., \& Oughton, S. 1995, Phys. Fluids, 7 , 2886

Howes, G. G., Cowley, S. C., Dorland, W., Hammett, G. W., Quataert, E., \& Schekochihin, A. A. 2008, J. Geophys. Res., 113, A05103

Iroshnikov, P. 1963, Astron. Zh. 40, 742

Kraichnan, R. H. 1965, Phys. Fluids 8, 1385

Li, X., \& Habbal, S. R. 2001, J. Geophys. Res., 106, 10669

Lithwick, Y., \& Goldreich, P. 2003, ApJ, 582, 1220

Lithwick, Y., Goldreich, P., \& Sridhar, S. 2007, ApJ, 655, 269

Malara, F., Primavera, L., \& Veltri, P. 2000, Phys. Plasmas, 7, 2866

Malara, F., Primavera, L., \& Veltri, P., 2001, in Recent Insights into the Physics of the Sun and Heliosphere: Highlights from SOHO and Other Space Missions, eds. Brekke, P., Fleck, B., \& Gurman, J.B., Proceedings of the 24th General Assembly of the IAU (IAU Symposium 203, pp. 511-513), (San Francisco: Astronomical Society of the Pacific)

Matthaeus, W. H., \& Montgomery, D. 1980, New York Acad. Sci., 357, 203

Matthaeus, W. H., Zank, G. P., Oughton, S., Mullan, D. J., \& Dmitruk, P. 1999, ApJL, 523, L93

Narayan, R., \& Medvedev, M. 2001, ApJ, 562, 129

Ng, C. S., \& Bhattacharjee, A. 1997, Phys. Plasmas, 4, 605

Ng, C. S., Bhattacharjee, A., Isenberg, P. A., Munsi, D., \& Smith, C. W., Kolmogorov Versus Iroshnikov-Kraichnan Spectra: Consequences for Ion Heating in the Solar Wind, in preparation.

Perez, J. C., \& Boldyrev, S. 2009, Phys. Rev. Lett., 102, 025003 (2009)

Podesta, J., \& Bhattacharjee, A., Phys. Rev. Lett., submitted 
Pouquet, A., Sulem, P. L., \& Meneguzzi, M. 1988, Phys. Fluids, 31, 2635

Roberts, D. A., Goldstein, M. L., Klein, L. W., \& Matthaeus, W. H. 1987, J. Geophys. Res., 92, 12023

Roberts, D. A., Goldstein, M. L., Matthaeus, W. H., \& Ghosh, S. 1992, J. Geophys. Res., 97, 17115

Sagdeev, R. Z., \& Galeev, A. A. 1969, Nonlinear Plasma Theory (Benjamin: New York)

Schekochihin, A. A., Cowley, S. C., Dorland, W., Hammett, G. W., Howes, G. G., Quataert, E., \& Tatsuno, T 2009, ApJS, accepted

Smith, C. W. 2003, Solar Wind Ten: Proceedings of the Tenth International Solar Wind Conference, AIP Conference Proceedings, 679, 413

Tu, C. Y., \& Marsch, E., 1995, Sp. Sci. Rev., 73, 1

Velli, M. 1993, A\&A, 270, 304

Verdini, A., \& Velli, M. 2007, ApJ, 662, 669

Viñas, A.F. \& Goldstein, M.L. 1991, J. Plasma Phys., 46, 129

Yan, H., \& Lazarian, A. 2004, ApJ, 614, 757 